\title{
Wanted: A FRAME for Staging Functional Decline in Older Adults
}

$\mathrm{C}$ linicians are accustomed to staging diseases in fours. The examples abound: the New York Heart Association Class I to IV categories for heart failure, the Global Initiative for Chronic Obstructive Lung Disease (GOLD), the four-stage system used for most cancers - all ranging from mild disease with potential for reversibility in Stage 1 to gravity and irreversibility in Stage 4. Staging systems provide clinicians with a useful framework to divide patients into clinically homogeneous groups for the purposes of prognosis and selection of treatments.

One of the most vexing problems today in caring for older adults is the lack of a widely recognized model for categorizing older adults with functional decline that helps inform prognosis and guide treatment. This challenge of developing a universal staging system is more difficult in older adults because of the accumulation of multiple chronic health conditions and variation in the trajectories of decline associated with different conditions. Occasionally, one condition dominates the picture because of its severity (e.g., metastatic cancer, Class IV heart failure), but more often this is not case, as in individuals with multiple moderately severe chronic conditions. When multiple conditions coexist, the potential for additive or interactive effects of these health conditions is greater, further complicating determination of risk. The current method of counting number of health conditions is a common but inadequate substitute for current function and potential for functional recovery (or decline). All clinicians have patients with long lists of health problems who are still leading active lives while other patients with a similar (or shorter) list of health problems seem to be on the verge of rapidly declining. What objective ways can one be distinguished from the other? Certain progress has been made in global measures of decline for older adults, ${ }^{1-3}$ but a clinically useful four-stage measure is still elusive.

When grouping participants in intervention studies, as in meta-analyses, the need for a globally accepted functional decline measure is also imperative. In this journal, Dr. Lin and colleagues ${ }^{4}$ report the challenges they faced when reviewing the evidence on multifactorial interventions to prevent functional decline in older adults for the U.S. Preventive Services Task Force. The lack of a widely recognized model for categorizing older adults into homogeneous groups greatly limits the interpretation of the data.

DOI: $10.1111 / \mathrm{j} .1532-5415.2012 .04215 . \mathrm{x}$
Older adults vary in their stage of functional decline. Some older adults may have declined only modestly since their peak function in the third or fourth decades; they could be considered "Stage 1." A Stage 1 individual might be counseled to stabilize his or her function with regular exercise. Other older adults will have had greater declines. Such differences are probably also related to future risk of further decline and death, ${ }^{5-7}$ with comorbidities probably contributing to moderate (Stage 2) and severe (Stage 3) risk. Such individuals might benefit greatly from the specialized multiprong interventions that Lin and colleagues review. Last, the worst stage would be Stage 4, analogous to other staging systems in its gravity and irreversibility; patients in this stage have declined too far for even a well-designed multifactorial intervention to have much effect.

One reason it has not been possible to find clinically significant pooled effects of functional decline interventions is because of mixing of baseline staging. The relationship between baseline risk and effectiveness is true for most healthcare interventions, with higher-risk patients benefiting more. For example, comparing the benefit of the identical intervention of daily aspirin, prevention of coronary events is less effective in studies of primary prevention (low risk) than studies of secondary prevention (high risk). It is likely that the small pooled effect that Lin and colleagues found is due to mixing low- and high-risk patients between studies that varied in the level of risk of their target populations and that, within studies, participants also varied in their baseline risk. Furthermore, because how to identify those in Stage 4 global functional decline is not yet understood, it is possible that inclusion of participants in Stage 4 may have also contributed to not finding a link between intervention and benefit. With single-disease clinical interventions, individuals in Stage 4 do not receive treatments studied in previous stages; for example, aspirin therapy has not been studied as an intervention for individuals with end-stage coronary disease on a balloon pump.

Geriatric medicine needs a model of functional decline staging in older adults. A number of measures have been reviewed in Lin and colleagues, but no single model has risen to the top in terms of widespread acceptance and use (e.g., at the level of the New York Heart Association classification for heart failure). We believe that the critical factors of such a widely accepted model-let's call it the Functional Reserve Assessment Map for Elders, or FRAME—should be: 
Easy to apply in clinical practice and clinical interventions.

Based on clinical or administrative data or is potentially accessible through electronic medical records.

Based primarily on functional status.

Capture whether an individual has a severe, dominant, index health condition, as opposed to multiple chronic conditions that are also likely to contribute to probability of (or lack of) successful intervention beyond functional status.

The development and refinement of existing classification schemes for cancer, heart disease, rheumatoid arthritis, and chronic obstructive pulmonary disease took years of effort. A similar effort is needed now to develop the FRAME staging system for functional decline in older adults. It will not be easy, but without such a staging system, we will continue to be frustrated in our ability to identify interventions to help slow functional decline, to the detriment of millions of current and future older adults, at some point including ourselves.

Lillian Min, MD, MSHS

University of Michigan Medical School Ann Arbor Veterans Affairs Medical Center Ann Arbor, Michigan

Paul Shekelle MD, PhD

West Los Angeles Veterans Affairs Medical Center Los Angeles, California and

RAND Corporation-Health, Santa Monica, California

\section{ACKNOWLEDGMENTS}

Conflict of Interest: The editor in chief has reviewed the conflict of interest checklist provided by the authors and has determined that the authors have no financial or any other kind of personal conflicts with this paper.

Author Contributions: Both authors contributed equally to conception and design, preparation and revision, and approval of the final draft of this commentary.

Sponsor's Role: There is no sponsor for this work.

\section{REFERENCES}

1. Rockwood K, Mitnitski A. Frailty in relation to the accumulation of deficits. J Gerontol A Biol Sci Med Sci 2007;62A:722-727.

2. Stineman MG, Xie D, Pan Q et al. Activity of daily living staging, chronic health conditions, and perceived lack of home accessibility features for elderly people living in the community. J Am Geriatr Soc 2011;59:454-462.

3. Fried LP, Tangen CM, Walston J et al. Frailty in older adults: Evidence for a phenotype. J Gerontol A Biol Sci Med Sci 2001;56A:M146-M156.

4. Lin JS, Whitlock EP, Eckstrom E et al. Challenges in synthesizing and interpreting the evidence from a systematic review of multifactorial interventions to prevent functional decline in older adults. J Am Geriatr Soc 2012;60: 2157-2166.

5. Saliba D, Elliott M, Rubenstein LZ et al. The Vulnerable Elders Survey: A tool for identifying vulnerable older people in the community. J Am Geriatr Soc 2001;49:1691-1699.

6. Min LC, Elliott MN, Wenger NS et al. Higher Vulnerable Elders Survey scores predict death and functional decline in vulnerable older people. J Am Geriatr Soc 2006;54:507-511.

7. Min L, Yoon W, Mariano J et al. The Vulnerable Elders-13 Survey predicts 5 -year functional decline and mortality outcomes in older ambulatory care patients. J Am Geriatr Soc 2009;57:2070-2076. 